\title{
Adsorption of a Synthetic Rubber onto a White Carbon Surface
}

\author{
Masami Kawaguchi, Tatsuya SANo, and Akira TAKahashi \\ Department of Industrial Chemistry, Faculty of Engineering, \\ Mie University, 1515 Kamihamacho, Tsu, Mie 514, Japan.
}

(Received April 8, 1981)

\begin{abstract}
Adsorption of a synthetic rubber, polybutadiene onto a white carbon surface, i.e., nonporous silica (Aerosil 130), from cyclohexane solutions at $35^{\circ} \mathrm{C}$ was studied by IR spectroscopy and differential refractometry. The observed infrared frequency shifts of the silanol groups for 1,7-octadiene and for a mixture of cis- and trans-2-octene, which are the monomeric analogues of polybutadiene, and also for polybutadiene, are attributed to the interaction between the silanol groups of the Aerosil 130 silica and the double bonds of these compounds. Adsorbance $(A)$ was determined using a differential refractometer, while both the fraction of the surface sites occupied $(\theta)$ and the fraction of the adsorbed segments per polymer chain $(p)$ were determined by IR spectroscopy. The experimental values of $A, \theta$, and $p$ observed in the plateau region of the adsorption isotherms were independent of molecular weight. The data were compared with the theory of Roe formulated for a lattice model. There was qualitative agreement between the observed data and Roe's theory.

KEY WORDS Adsorption / Synthetic Rubber / Polybutadiene / White Carbon / Silica / IR Spectroscopy / Frequency Shift / Double Bond / Silanol Group /
\end{abstract}

In the 1940s and 1950s, the adsorption of natural rubber $^{1}$ and synthetic rubbers such as polyisoprene and styrene-butadiene rubber (SBR) ${ }^{2-9}$ onto carbon blacks were extensively studied in relation to the development of rubber technology. As a result, it was found that adsorption of rubbers is controlled mainly by the interaction between the double bonds in rubber and the active sites on the carbon black surface. However, a fully adequate characterization of the carbon black surface used as the adsorbent was not possible at that time, the information being limited to only the adsorbance, which is expressed as the weight of adsorbed polymer per weight of the adsorbent. This retarded the quantitative interpretation of the interaction between the double bonds in rubber and surfaces.

Theories of polymer adsorption have been developed, and several important quantities such as adsorbed thickness $t$, adsorbance $A$, the fraction of the surface sites occupied $\theta$, and the fraction of the adsorbed segment per polymer, $p$, can be calculated as functions of molecular weight, polymer concentration, and the polymer-solvent, polymer- substrate, and solvent-substrate interaction parameters. ${ }^{10-13}$

If a well characterized substrate such as silica particles, for which the number of surface sites is known, is used as an adsorbent, a quantitative theoretical check for the values of $A, \theta$, and $p$ can be made. Thus, in a previous paper, ${ }^{14}$ we used the Aerosil 130 as a substrate and measured $A, \theta$, and $p$ for the adsorption of polystyrene onto the silica from cyclohexane solution at the theta temperature, and compared the observed values with the theories of Silberberg ${ }^{10}$ and of Scheutjens and Fleer. ${ }^{14}$

From a practical standpoint, instead of carbon blacks, silica particles have sometimes been used as a reinforcing material for elastomers. The name "white carbon" has been used in common for these particles. Botham and Thies ${ }^{15}$ have studied the adsorption of polybutadiene onto a silica surface from perchloroethylene solutions, but, limited the measurement to only the adsorbance and made no comparison with theories. In this paper, a well characterized Aerosil silica 130 was used as an adsorbent and adsorption of polybutadiene, a typi- 
Table I. Characteristics of polybutadienes

\begin{tabular}{|c|c|c|c|c|c|}
\hline \multirow{3}{*}{ Samples } & \multirow{3}{*}{$\frac{M_{w} \times 10^{-3}}{\mathrm{~g} \mathrm{~mol}^{-1}}$} & \multirow{3}{*}{$\frac{[\eta]}{\mathrm{dl} \mathrm{g}^{-1}}$} & \multicolumn{3}{|c|}{ Contents of } \\
\hline & & & $1-4$ cis & 1-4 trans & $1-2$ additive \\
\hline & & & $\%$ & $\%$ & $\%$ \\
\hline PBR-1 & 126 & 1.86 & 55 & 34 & 11 \\
\hline PBR-2 & 173 & 2.39 & 56 & 34 & 10 \\
\hline PBR-3 & 241 & 3.01 & 58 & 32 & 10 \\
\hline PBR-4 & 314 & 3.69 & 51 & 38 & 11 \\
\hline
\end{tabular}

cal synthetic rubber, from cyclohexane solutions at $35^{\circ} \mathrm{C}$ was examined by using IR spectroscopy and differencial refractometry to clarify the nature of the interaction between rubbers and silica surface. A frequency shift in the silanol groups of the Aerosil silica caused by the interaction between the silanol groups and the double bond of polybutadiene was observed, and the $\theta$ and $p$ values were determined by IR spectroscopy. The data were compared with the theory of Roe. ${ }^{11}$

\section{EXPERIMENTAL}

\section{Material}

Polybutadiene manufactured by Asahi Chemical Industry Co. as NF-55A was fractionated at $25^{\circ} \mathrm{C}$ into nine fractions using isobutylacetate as a solvent and methanol as a precipitant. The fractionated samples were freeze-dried from benzene solutions. Their molecular weights were determined by gel permeation chromatography. Intrinsic viscosities of polybutadienes in cyclohexane at $35^{\circ} \mathrm{C}$ were measured by a Ubbelohde viscometer. The content of 14 cis, 1-4 trnas, and 1-2 additive in each fraction of polybutadiene was determined from the IR spectra of films prepared by casting benzene solutions onto a glass plate. The bands of particular interest were located at $967 \mathrm{~cm}^{-1}$ (1-4 trans), $911 \mathrm{~cm}^{-1}(1-2$ additive), and $724 \mathrm{~cm}^{-1}$ (1-4 cis), respectively. Extinction coefficients determined by Binder ${ }^{16}$ were employed for the quantitative determination of 1-4 trans, 1-2 additive, and 1-4 cis contents. The characteristics of the polybutadiene samples are given in Table I.

Cyclohexane was purified by the previously described method. ${ }^{14}$ Extra pure grade 1,7-octadiene and a mixture of cis- and trans-2-octene were used without further purification.
The nonporous Aerosil 130 silica supplied by Degussa A. G. (West Germany) were used as the adsorbent after cleaning it by the procedure described in a previous paper. ${ }^{14}$ The concentration of surface silanol groups was determined by the method of Boehm and Schneider ${ }^{17}$ to be three silanol groups per $100 \mathrm{~A}^{2}$. According to the manufacturer, the particle diameter was $160 \mathrm{~A}$ with a surface area of $141 \mathrm{~m}^{2} \mathrm{~g}^{-1}$.

\section{Adsorption of Polybutadienes onto the Silica}

Twenty-five $\mathrm{ml}$ of polybutadiene cyclohexane solution of known concentration were mixed with the silica (usually about $0.025 \mathrm{~g}$ ) in a stoppered centrifuge tube and the mixture was gently stirred by a magnetic chip for $20 \mathrm{~h}$ at $35^{\circ} \mathrm{C}$. The silica suspensions were centrifuged at $5,000 \mathrm{~g}$ for $5 \mathrm{~min}$ to sediment the silica and then the supernatant was carefully removed. The concentration of polybutadiene $C_{\mathrm{p}}$ in the supernatant was measured by a Wood differential refractometer at a wavelength of $546 \mathrm{~nm}$. The relevent concentration of polybutadiene was determined from a calibration curve. Adsorbance ( $\mathrm{g} \mathrm{cm}^{-2}$ of silica), $A$, was determined from the difference between the amount of the polybutadiene initially added and that in the supernatant, and also from the amount of the silica added.

The method for IR measurements on the sedimented silica and the silica immersed in 1,7 octadiene and also in 2-octene was similar as described previously. ${ }^{14}$

The method for calculating for the fraction of the surface sites occupied, $\theta$, and for the fraction of segments adsorbed, $p$ are described in a previous paper. $^{14}$ 


\section{RESULTS}

\section{Adsorbance}

Adsorption isotherms of the samples studied are shown in Figure 1. Initially, the adsorption isotherms rose steeply with increasing equilibrium polymer concentration and reached a plateau region. These isotherms are of the high affinity type, showing that the adsorbance at a bulk equilibrium polymer concentration of $0.1 \mathrm{~g} / 100 \mathrm{ml}$ is well in the plateau region for all samples. The adsorbances are summarized in Table II for all the samples. The adsorbance in the plateau region was almost constant and independent of molecular weight.

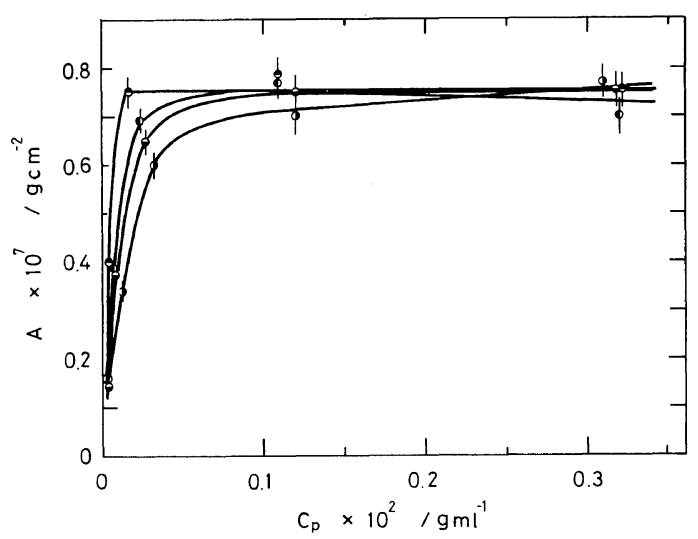

Figure 1. Adsorption isotherms of polybutadiene: $\boldsymbol{O}$, PBR-1; ๑, PBR-2; D, PBR-3; ๑, PBR-4.

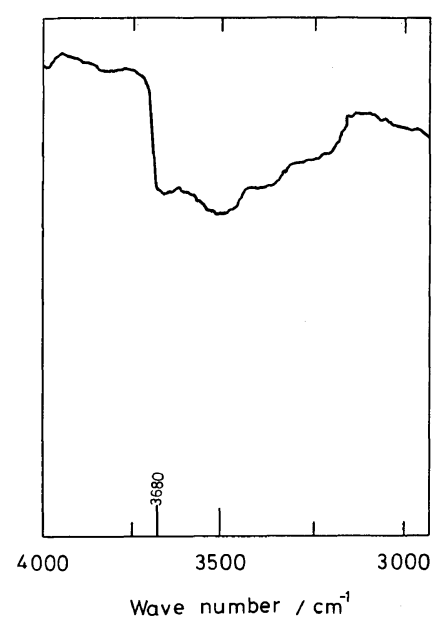

Figure 2. Infrared spectrum of Aerosil 130 in cyclohexane, in the presence of polybutadiene of PBR-2.

\section{Fraction of Surface Sites Occupied, $\theta$}

The differential infrared spectrum between the supernatant polybutadiene solution and the silica on which polybutadiene was adsorbed, shows two characteristic bands in Figure 2; one band located at $3,680 \mathrm{~cm}^{-1}$ is assigned to the isolated silanol groups and the other located at $3,500 \mathrm{~cm}^{-1}$, is assigned to the attached double bond of polybutadiene, i.e., the $-\mathrm{CH}=\mathrm{CH}-$ group attached to the isolated silanol groups. Thus, both the number of free silanol groups and the number of the occupied silanol groups can be determined, provided the extinction coefficients for the respective groups are known. For the free silanol groups, ${ }^{14}$ the extinction coefficient at $3,680 \mathrm{~cm}^{-1}$ was determined to be 55.6 $1 \mathrm{~mol}^{-1} \mathrm{~cm}^{-1}$.

The IR spectra of the silica immersed in 1,7 octadiene and 2-octene, which are considered to be model compounds to the monomer unit of polybutadiene because of their double bonds, are shown in Figure 3. The observed absorbance at $3,375 \mathrm{~cm}^{-1}$ can be attributed to the interaction between the double bonds in these model compounds and the silanol groups on the silica surface. The shift of the silanol groups due to the model compounds is greater than that due to polybutadiene, possibly because of the difference in the strength of the interaction between the silanol groups and the double bonds in the model compounds and polybutadiene as well. However, we have assumed that the extinction coefficient, $1341 \mathrm{~mol}^{-1} \mathrm{~cm}^{-1}$ at

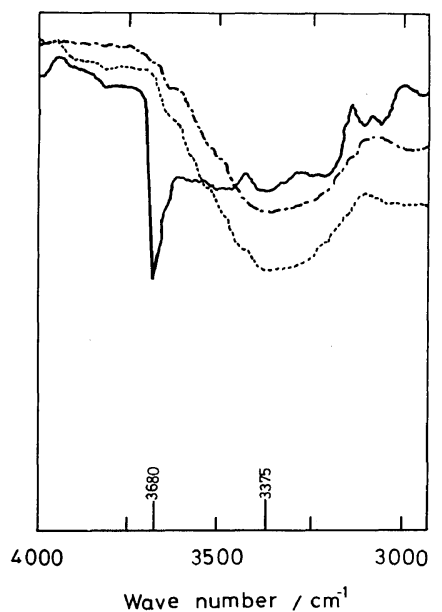

Figure 3. Infrared spectra of Aerosil 130 immersed in $(-)$ cyclohexane, (----) 1,7-octadiene, and (-2-octene. 
$3,500 \mathrm{~cm}^{-1}$ of the double bond in polybutadiene is equal to that of the model compounds at 3,375 $\mathrm{cm}^{-1}$

The fraction of the surface sites occupied, $\theta$, can be calculated from the following equation,

$$
\theta=S_{3,500} /\left(S_{3,500}+S_{3,680}\right)
$$

where $S_{3,680}$ and $S_{3,500}$ are the number of free sites and the occupied sites by double bonds, respectively. The value of $\theta$, calculated from eq 1 are listed in Table II. These values increase with increasing bulk equilibrium polymer concentration for each molecular weight. The limiting value of $\theta$ in the plateau region is 0.5 irrespecive of the molecular weight.

\section{Fraction of Segment Adsorbed, $p$}

The fraction of segments adsorbed, $p$, is summarized in Table II and decreases with increasing bulk equilibrium concentration for each molecular weight. The value of $p$ in the plateau region is almost constant, being 0.2 for all the samples.

\section{DISCUSSION}

\section{Frequency Shift of Silanol Groups Attached by Double Bonds}

Strong infrared frequency shifts of the silanol groups were observed for the silica immersed in 1,7octadiene and 2-octene which are analogous compounds of a monomer unit of polybutadiene. These shifts can be attributed to the interaction between their double bonds and the silanol groups, since Little and Mathieu ${ }^{18}$ previously observed by IR spectroscopy similar shifts of the silanol groups when $\mathrm{CH}_{2}=\mathrm{CH}_{2}$ was allowed to adsorb on the silanol groups. The similar infrared spectroscopic shift observed for adsorbed polybutadiene is thus explained by the interaction between the double bonds of polybutadiene and the silanol groups. Moreover, with 1-octadecene, a similar frequency shift of the silanol was observed by Erkelens and

Table II. Adsorption data of polybutadienes on Aerosil 130

\begin{tabular}{|c|c|c|c|c|c|c|}
\hline \multirow{3}{*}{ Samples } & \multirow{3}{*}{$\begin{array}{c}\begin{array}{c}\text { Bulk } \\
\text { equilibrium } \\
\text { concentration }\end{array} \\
C_{\mathrm{p}} \times 10^{2} / \mathrm{g} \mathrm{ml}^{-1}\end{array}$} & \multirow{3}{*}{$\frac{\text { Adsorbance }}{A \times 10^{7} / \mathrm{g} \mathrm{cm}^{2}}$} & \multirow{3}{*}{$\begin{array}{l}\text { Fraction of } \\
\text { segments } \\
\text { adsorbed } \\
p\end{array}$} & \multirow{3}{*}{$\begin{array}{c}\text { Fraction of } \\
\text { surface } \\
\text { sites occupied } \\
\theta\end{array}$} & \multicolumn{2}{|c|}{ Surface excess } \\
\hline & & & & & $\Gamma$ & $\Gamma=\theta / p$ \\
\hline & & & & & & \\
\hline \multirow{5}{*}{ PBR-1 } & 0.0015 & $0.16 \pm 0.01$ & $0.77 \pm 0.08$ & $0.34 \pm 0.03$ & $0.61 \pm 0.03$ & $0.44 \pm 0.04$ \\
\hline & 0.012 & $0.34 \pm 0.02$ & $0.36 \pm 0.04$ & $0.44 \pm 0.04$ & $1.25 \pm 0.06$ & $1.22 \pm 0.12$ \\
\hline & 0.032 & $0.60 \pm 0.03$ & $0.22 \pm 0.02$ & $0.47 \pm 0.05$ & $2.23 \pm 0.11$ & $2.17 \pm 0.22$ \\
\hline & 0.12 & $0.70 \pm 0.04$ & $0.21 \pm 0.02$ & $0.51 \pm 0.05$ & $2.60 \pm 0.13$ & $2.43 \pm 0.24$ \\
\hline & 0.31 & $0.77 \pm 0.04$ & $0.18 \pm 0.02$ & $0.53 \pm 0.05$ & $2.87 \pm 0.14$ & $2.95 \pm 0.29$ \\
\hline \multirow{5}{*}{ PBR-2 } & 0.0038 & $0.14 \pm 0.01$ & $0.78 \pm 0.08$ & $0.36 \pm 0.04$ & $0.53 \pm 0.03$ & $0.46 \pm 0.05$ \\
\hline & 0.0083 & $0.37 \pm 0.02$ & $0.37 \pm 0.04$ & $0.48 \pm 0.05$ & $1.37 \pm 0.07$ & $1.28 \pm 0.13$ \\
\hline & 0.027 & $0.65 \pm 0.03$ & $0.23 \pm 0.02$ & $0.53 \pm 0.05$ & $2.40 \pm 0.12$ & $2.36 \pm 0.24$ \\
\hline & 0.12 & $0.75 \pm 0.04$ & $0.19 \pm 0.02$ & $0.53 \pm 0.05$ & $2.76 \pm 0.14$ & $2.77 \pm 0.28$ \\
\hline & 0.32 & $0.75 \pm 0.04$ & $0.16 \pm 0.02$ & $0.49 \pm 0.05$ & $2.79 \pm 0.14$ & $3.10 \pm 0.31$ \\
\hline \multirow{5}{*}{ PBR-3 } & 0.0023 & $0.16 \pm 0.01$ & $0.70 \pm 0.07$ & $0.35 \pm 0.04$ & $0.58 \pm 0.03$ & $0.50 \pm 0.05$ \\
\hline & 0.0060 & $0.39 \pm 0.02$ & $0.27 \pm 0.03$ & $0.46 \pm 0.05$ & $1.45 \pm 0.07$ & $1.67 \pm 0.17$ \\
\hline & 0.023 & $0.69 \pm 0.03$ & $0.21 \pm 0.02$ & $0.48 \pm 0.05$ & $2.55 \pm 0.13$ & $2.23 \pm 0.22$ \\
\hline & 0.11 & $0.77 \pm 0.04$ & $0.17 \pm 0.02$ & $0.50 \pm 0.05$ & $2.84 \pm 0.14$ & $2.88 \pm 0.29$ \\
\hline & 0.32 & $0.70 \pm 0.04$ & $0.18 \pm 0.02$ & $0.49 \pm 0.05$ & $2.60 \pm 0.13$ & $2.70 \pm 0.27$ \\
\hline \multirow{5}{*}{ PBR-4 } & 0.0023 & $0.16 \pm 0.01$ & $0.67 \pm 0.07$ & $0.35 \pm 0.04$ & $0.58 \pm 0.03$ & $0.53 \pm 0.05$ \\
\hline & 0.0045 & $0.40 \pm 0.02$ & $0.39 \pm 0.04$ & $0.45 \pm 0.05$ & $1.50 \pm 0.08$ & $1.16 \pm 0.12$ \\
\hline & 0.016 & $0.75 \pm 0.04$ & $0.20 \pm 0.02$ & $0.50 \pm 0.05$ & $2.76 \pm 0.14$ & $2.51 \pm 0.25$ \\
\hline & 0.11 & $0.79 \pm 0.04$ & $0.23 \pm 0.02$ & $0.51 \pm 0.05$ & $2.92 \pm 0.15$ & $2.22 \pm 0.22$ \\
\hline & 0.32 & $0.75 \pm 0.04$ & $0.19 \pm 0.02$ & $0.51 \pm 0.05$ & $2.76 \pm 0.14$ & $2.61 \pm 0.26$ \\
\hline
\end{tabular}


Liefkens. ${ }^{19}$ The present experimental data are in agreement with their results. When the observed small frequency shift of silanol groups adsorbed by polybutadiene is compared with the large frequency shift due to the model compounds, it seems likely that the interaction with the surface silanol groups is weaker for the double bond of polybutadiene than for that of the model compounds. Polybutadiene has 1-4 trans, 1-4 cis, and 1-2 additive structures, and this structural irregularity may be responsible for this difference.

\section{Surface Excess $\Gamma$}

The surface excess, $\Gamma$, the total number of molecular segments adsorbed per lattice site, is an important 'quantity, since it can be regarded as the adsorbed amount per lattice site when experimental data are compared with the theoretical treatment based on a lattice model. $\Gamma$ is defined as,

$$
\Gamma=\left(A N_{\mathrm{A}} / n_{\mathrm{OH}} M_{\mathrm{u}}\right)
$$

where $A$ is the adsorbance $\left(\mathrm{g} \mathrm{cm}^{-2}\right), N_{\mathrm{A}}$ is Avogadro's constant, $n_{\mathrm{OH}}$ is the number of silanol sites per unit area, being $3 \times 10^{14}$ in the present case, and $M_{\mathrm{u}}$ is the molecular weight of the butadiene monomer unit, $\theta$ is the fraction of surface sites occupied, and $p$ is the fraction of adsorbed segments. The values of $\Gamma$ calculated from measured adsorbance are compared with the values of $\theta / p$ in Table II. We find that the values of $\Gamma$ determined by the two methods are consistent.

\section{Comparison of Experimental Results and Theory}

Several theories for polymer adsorption are available. In Silberberg's theory, ${ }^{10}$ the loop-train conformation is assumed, while Scheutjens and Fleer's theory ${ }^{13}$ is based on the loop-train-tail conformation. In these theories, $p, \theta$, and $\Gamma$ are calculated as a function of Flory-Huggins parameter $\chi$. However, the published graphical results of these authors deal with only two cases: $\chi=0.5$ and $\chi=0$. The $\chi$ parameter for polybutadiene-cyclohexane at $35^{\circ} \mathrm{C}$ was determined to be 0.45 from thermodynamic analysis using the Stockmayer-Fixman plot with the intrinsic viscosity data in Table I. Hence, we were unable to compare at the present stage our results with the theories of Silberberg ${ }^{10}$ and Scheutjens and Fleer. ${ }^{13}$ As an alternative, we selected the more comprehensive theory of Roe ${ }^{11}$ since it allows $\Gamma$ to be evaluated easily as a function of $\chi$.
For this calculation, we used the computer program kindly furnished by Professor Roe.

In Roe's theory, the close-packed hexagonal lattice is assumed and the simplifying assumption was made such that each segment of a polymer chain makes the same contribution to the density of a segment at any distance from the surface. This theory specifies no particular conformations for adsorbed polymers. Although $p$ and $\theta$ are not explicitly considered in Roe's theory, the value of $p$ can be evaluated from the following equation,

$$
p=\psi_{2}{ }^{1} / \sum_{i=1}^{\infty} \psi_{2}{ }^{i}
$$

where $\psi_{2}{ }^{1}$ and $\psi_{2}{ }^{i}$ are the volume fractions of polymer in the first layer and in the $i$-th layer, respectively. The value of $\theta$ was calculated by multiplying $\Gamma$ by the calculated $p$ value. The three important quantities of $\Gamma, \theta$, and $p$ can be calculated as functions of polymer concentration, molecular weight, $\chi$ parameter, and polymer-surface interaction parameter, $\chi_{\mathrm{s}}$. Except for $\chi_{\mathrm{s}}$ the other quantities are already known. Thus, we first attempted to evaluate $\chi_{\mathrm{s}}$ so as to make a quantitative interpretation of the experimental data.

Since the molecular weight dependence of $\Gamma, \theta$, and $p$ was almost absent, we selected the sample PBR-2 with a molecular weight of $173 \times 10^{3}$ for comparison. In Figures 4, 5, and $6, \Gamma, p$, and $\theta$ for PBR-2 are compared with those calculated by the theory of Roe as a function of $\chi_{\mathrm{s}}$. We see that the calculated values of $\Gamma, p$, and $\theta$ increase with increasing $\chi_{\mathrm{s}}$, but above $\chi_{\mathrm{s}}=5.0, \Gamma, p$, and $\theta$ stay the same. As seen in Figure 6, the measured value of $\theta$ first sharply increases at the lower polymer concentration and then becomes constant around $C_{\mathrm{p}}=0.03 \mathrm{~g} / 100 \mathrm{ml}$. The calculated value of $\theta$ also shows a steep increase at a very low polymer concentration (less than $C_{\mathrm{p}}=0.001 \mathrm{~g} / 100 \mathrm{ml}$ ) and becomes independent of polymer concentration at higher $C_{\mathbf{p}}$. The measured values of $\theta$ in the plateau region are in fair agreement with the calculated $\theta$ value for $\chi_{\mathrm{s}}=0.7$.

In Figures 4 and 5 , the observed $\Gamma$ first increases sharply and the observed $p$ decreases with increasing polymer concentration; these values then become constant. A closer inspection of these figures reveals that the calculated values of both $\Gamma$ and $p$ show similar behavior; however, for all values of $\chi_{\mathrm{s}}$, in the plateau region, the calculated $\Gamma$ are always 


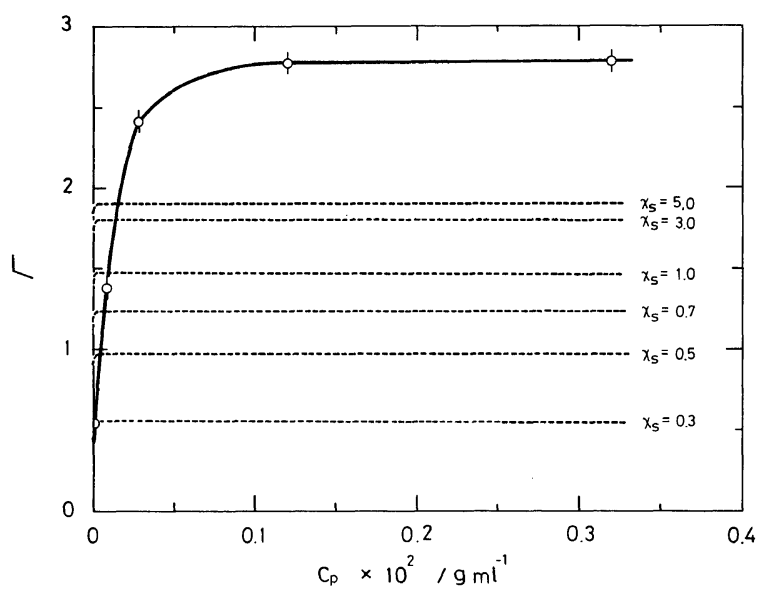

Figure 4. Plot of $\Gamma$ value vs. equilibrium polybutadiene concentration for PBR-2. The dashed lines were calculated by Roe's theory for different values of the polymer-surface interaction parameter, $\chi_{s}$.

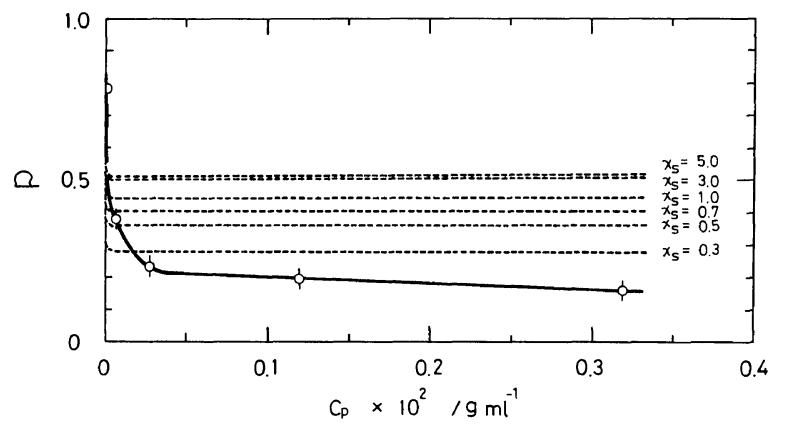

Figure 5. Plot of $p$ value $v s$. equilibrium polybutadiene concentration for PBR-2. The dashed lines were calculated by Roe's theory for different values of the polymer-surface interaction parameter, $\chi_{\mathrm{s}}$.

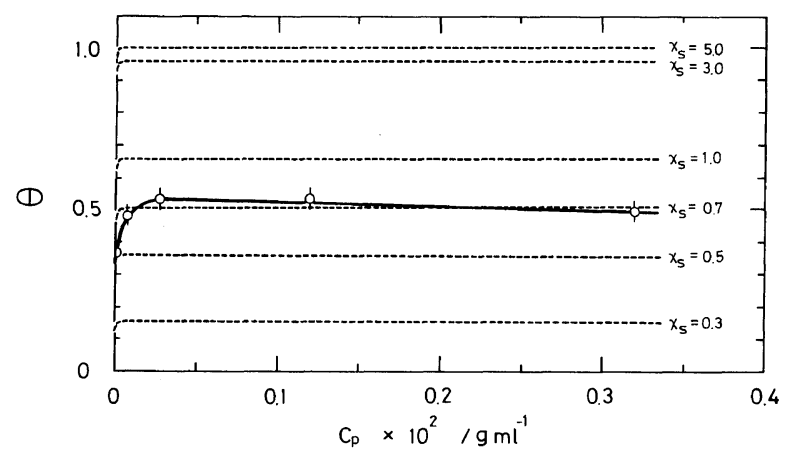

Figure 6. Plot of $\theta$ value vs. equilibrium polybutadiene concentration for PBR-2. The dashed lines were calculated by Roe's theory for different values of the polymer-surface interaction parameter, $\chi_{\mathrm{s}}$.

smaller than these observed. The calculated $p$ values are always larger than the observed $p$. Moreover, for $\chi_{\mathrm{s}}=0.7$ the calculated value of $\Gamma$ is one half the measured value of 2.8 and the calculated value of $p$ is nearly twice as large as the observed $p$, as can be seen from Figures 4 and 5. If an extreme value of $\chi_{s}$, say 5.0 , is chosen, the calculated value of $\Gamma$ approaches the measured value, but no complete 
agreement between calculated and observed $\Gamma$ can be made for any $\chi_{s}$. Thus, there is not available any one value of $\chi_{\mathrm{s}}$ which can explain all three experimental quantities of $\Gamma, p$, and $\theta$ at the same time. Nevertheless, it is important that Roe's theory can at least qualitatively explain the concentration dependence of $\Gamma$, and $p$, and $\theta$. The discrepancies between theory and experiment may be due to the following two reasons. The first reason may be the geometry of the silica surface; the silica particles used here are small spheres and have large curvature, while a plane surface was assumed in the theory. The second reason is possibly the structure of the polybutadienes employed in this study, consisting of 1-4 trans, 1-4 cis, and 1-2 additive structures. These structures should thus be considered copolymers. Strictly speaking, Roe's theory is not really applicable to copolymers.

\section{CONCLUSION}

From the IR spectra of silica on which polybutadienes were adsorbed, it is concluded that the observed large frequency shift of the silanol groups was caused by interaction between the double bonds in polybutadiene and the surface silanol groups. This means that polybutadiene is tightly adsorbed on the silica surface of the silanol groups. The experimental dependence of $\theta, p$, and $\Gamma$ on polymer concentration was found to be in qualitative agreement with the theoretical predictions of Roe.

Acknowledgement. The authors are deeply grateful to Professor R. J. Roe for kindly offering them the use of his computer program. We are also indebted to Mr. H. Kakitani of Yokkaichi
Research Laboratory, Mitsubishi Monsanto Chemical Co. for supplying the polybutadiene samples.

\section{REFERENCES}

1. G. Krauss and J. Dugone, Ind. Eng. Chem., 47, 1809 (1955).

2. G. Goldfinger, Rubber Chem. Technol., 18, 286 (1945) and 19, 614 (1946).

3. I. Lander, C.R., 225, 629 (1947).

4. L. E. Ambroski, C. E. Black, and G. Goldfinger, Rubber Chem. Technol., 23, 417 and 803 (1950).

5. I. M. Kolthoff and A. Kahn, J. Phys. Chem., 54, 251 (1950).

6. I. M. Kolthoff, R. G. Gutmacher, and A. Kahn, $J$. Phys. Chem., 55, 1240 (1951).

7. I. M. Kolthoff and R. G. Gutmacher, J. Phys. Chem., 56, 740 (1952).

8. M. A. Golub, J. Polym. Sci., 11, 583 (1953).

9. J. S. Binford, Jr., and A. M. Gessler, J. Phys. Chem., 63, 1376 (1959).

10. A. Silberberg, J. Chem. Phys., 48, 2835 (1968).

11. R. J. Roe, J. Chem. Phys., 60, 4192 (1974).

12. M. Lal and R. J. T. Stepto, J. Polym. Sci., Polym. Symp., No. 61, 401 (1977).

13. J. M. H. M. Scheutjens and G. J. Fleer, J. Phys. Chem., 83, 1619 (1979).

14. M. Kawaguchi, K. Hayakawa, and A. Takahashi, Polym. J., 12, 265 (1980).

15. R. A. Botham and C. Thies, J. Colloid Interface Sci., 45, 512 (1973).

16. J. L. Binder, Anal. Chem., 26, 1877 (1954).

17. H. P. Boehm and M. Z. Schneider, Anorg. Allgem. Chem., 301, 326 (1959).

18. L. H. Little and M. V. Mathieu, Proceedings of the 2nd International Congress on Catalysis, Paris 1960 , p 771 .

19. J. Erkelens and Th. J. Liefkens, J. Catalyst, 39, 173 (1975). 